\title{
ANTIMICROBIAL AND PHYTOCHEMICAL PROPERTIES OF CRUDE EXTRACTS OF SOME SELECTED PLANTS AGAINST SOME MICROBIAL ISOLATES
}

\author{
Chukwudebe $^{1}$, E.P., Bankole, S.O ${ }^{* 2}$, Asonibare, A.O ${ }^{2}$ and Adedokun, S.A ${ }^{3}$ \\ ${ }^{1}$ Biomedicinal Research Centre, Forestry Research Institute of Nigeria, Jericho, Ibadan. \\ ${ }^{2}$ Biotechnology Section, Bioscience Department \\ ${ }^{3}$ Basic Science \& General Studies Department, Federal College of Forestry, Ibadan
}

http://doi.org/10.35410/IJAEB.2019.4428

\begin{abstract}
Antimicrobial and phytochemical properties of the crude extract of some selected plant samples against some microbial isolates was investigated.Plant samples were extracted using ethyl acetate and methanol as extracting solvents based on polarity. Antimicrobial studies was carried out using Agar disc diffusion method. Zones of inhibitions were measured, Tetracycline, Ampicillin, Chloramphenicol and Ofloxacin antibiotics were used as positive control. Extracting solvent served as negative control and work was done in triplicates.Plant samples were found to contain several bioactive compounds in various combinations. Ethyl acetate Pawpaw leaves extract gave the best results with zones of inhibition as high as $27.3 \pm 11.7 \mathrm{~mm}$ and $25.3 \pm 0.6 \mathrm{~mm}$ against Escherichia coli and Candida albicans respectively, while methanol Pawpaw leaves extracts gave the best result with zone of inhibition as high as $25.0 \pm 13.0 \mathrm{~mm}$ against Staphylococcus aureus. Ampicillin and Chloramphenicol in all cases and for both extracting solvents showed no significant difference in the mean zone of inhibition compared with plant extracts at $5 \%$ level of significance. The minimum bactericidal concentration was found with ethyl acetate pawpaw leaves extract against Escherichia coli and Pseudomonas aeruginosa (bactericidal) at 100mg/ml and $200 \mathrm{mg} / \mathrm{ml}$ respectively while all the others were bacteriostatic.
\end{abstract}

It was thus concluded that the selected plant extracts could serve as alternative drugs for the treatment of diseases.

Keywords: Bacteriostatic, Extract, Inhibition,Phytochemical.

\section{INTRODUCTION}

Indigenous knowledge of herbal medicine is a big source of the modern knowledge (Kakaretal., 2012). Herbal plants and their preparations have been reported for antimicrobial, antimalarial, anti-inflammatory, anti-parasitic, anti-diabetic, anthelmintic, anti-obesity, anti-cancer and antiviral activities (Nimrietal., 1999).CaricapapayaLinn(Pawpaw), belonging to family Caricaceae is commonly known as papaya in English is commonly known for its food and nutritional values throughout the world. The medicinal properties of papaya fruit and other parts of the plant are also well known in traditional system of medicine. Each part of papaya tree possesses economic value when it is grown on a commercial scale (Krishna et al., 2008). Traditionally, leaves have 
been used for treatment of a wide range of ailments, like in treatment of malaria, dengue, and jaundice, immune modulatory and antiviral activity amongst others.According to Ayoola and Adeyeye (2010), the phytochemical analysis of the leaves of Carica papayashowed the presence of saponins, cardiac glycosides, and alkaloids. Tannin was absent in the leaves. The presence of saponins supports the fact that pawpaw leaf has cytotoxic effects such as permealization of the intestine, as saponins are cytotoxic (Okwu and Okwu, 2004). Vijayakumar et al., (2015) in their study using the extracts prepared from leaf (acetone, aqueous, ethanol and methanol) of Carica papaya, found the presence of Alkaloids, Flavonoids, phenols, Saponins, and Sterols, also it exhibited highest antimicrobial activity against pathogenic microbes bacteria and fungi.

Yogiraj et al., (2014) in their report on the antibacterial and antifungal ability of both fresh and dried leaves of Carica papaya against bacteria and fungi of medical importance using the disc diffusion method reported very significant broad spectrum antimicrobial activity against Gramnegative and Gram-positive bacteria and fungi.

Mangifera indica (Mango) is a large evergreen tree, with a heavy, dome-shaped crown. It belongs to the family Anacardiaceae.It is found all over the tropical regions of the world where it is used as a horticultural and medicinal plant.Various parts of the plant are used as a dentrifrice, antiseptic, astringent, diaphoretic, stomachic, vermifuge, tonic, laxative and diuretic and to treat diarrhea, dysentery, anaemia, asthma, bronchitis, cough, hypertension, insomnia, rheumatism, toothache, leucorrhoea, haemorrhage and pile. The leaves have been reported to contain saponins, glycosides, unsaturated sterols, polyphenols, euxanthin acid, mangiferine, mangin, gallic tannins, etc. The ashes of the leaves are used to treat burns, scalds, sores, cough and diarrhoea in South America and other parts of the world (Dweck, 2001; Hirte, 2002).According to Okwu and Ezenagu (2008), in their evaluation of the phytochemical composition of mango (Mangiferaindicalinn), the stem, bark and leaves showed presence of tannins, saponnins, alkaloids and flavonoids. According toNwankwo and Osaro-Mathew (2014), it was stated that the ethanolic extract of $M$. indica had mild inhibitory effects on Staphylococcus aureus and Escherichia coli while the hot water extract of the same concentration showed no inhibitory effects on these organisms.Mary et al., (2013) in her work onphytochemical analysis and anticancer activity of leaf extract ofmangiferaindica stated that it had antimicrobial activity against nine bacteria.According to the research, the presence of phyto-constituents in the leaf extracts may be responsible for these antibacterial activity of the plant.

\section{MATERIALS AND METHODS}

Collection of Plant Parts: Leaves, as well as Ripe and Unripe fruit peel of Mangifera indica (Mango) and Carica papaya (paw paw) were collected from FRIN Herbal garden. They were identified and authenticated at the taxonomy department of the institute. Samples collected were allowed to dry at room temperature $\left(35^{\circ} \mathrm{C}\right)$ before milling into powered form. They were kept in tight containers for further use.

Collection and Isolation of Test Microorganisms: Clinical isolates were collected. The four isolates used in this study were Staphylococcus aureus, Escherichia coli, Candida albicans and Pseudomonas aeruginosa. These were obtained from University of Ilorin teaching Hospital, Kwara State, Ilorin. They were sub-cultured and maintained in Nutrient agar and Potato dextrose agar slant for bacteria and fungi respectively. 
Preparation of Inoculum: A loopful of test organism was taken from agar slants into test tubes containing nutrient broth and Potato dextrose liquid. The test tubes was then incubated for 24hours at $37^{\circ} \mathrm{C}$ and 48 hours at $30^{\circ} \mathrm{C}$. The isolates in the broth was standardized using $0.5 \mathrm{ml}$ Mcfarlands turbidity standard. This was done by adding $0.5 \mathrm{ml}$ of $1.175 \%$ of anhydrous Barium chloride drop-wise to $99.5 \mathrm{ml}$ of $1 \%$ sulphuric acid in $100 \mathrm{ml}$ volumetric flask and constantly swirling. This was mixed for 3-5 minutes until solution appeared homogeneous and free of clumps. Optical density of solution was examined using a spectrophotometer and wavelength of $625 \mathrm{~nm}$ was taken which is the accepted range for Mcfarland $0.5 \mathrm{ml}$. This was then dispensed into a glass screw cap tube and sealed with paraffin and then stored at room temperature until needed.

Extraction of Plant Materials: 200grams of milled plant parts were soaked in $1000 \mathrm{ml}$ of ethylacetate (extracting solvent). This was stirred with a glass rod and left in the shaker for 12 hours. It was then filtered using whatsman filter paper. The solution was then passed through the Rotary evaporator. Filtrate was left in the dessicator for future use. Similar procedure was also employed using methanol as extracting solvent.

Phytochemical Screening of Various Extracts of Plant Sample: This will be performed using the method described by Nweze et al., (2004) and Senthilkumar and Reetha (2009).The samples were screened for cardiac glycosides, alkaloids, flavonoids, triterpenesand steroids, anthocyanin and betacyanin, phenols. Tannins, saponin, glycosides, and anthraquinones.

Antimicrobial Sensitivity testing of the Extract on the Clinical isolates: Using agar disc diffusion techniques as described by Kirby-Bauer technique (Bauer et al., 1966; John and James, 1999), various concentration of plant extract $(400 \mathrm{mg} / \mathrm{ml}, 200 \mathrm{mg} / \mathrm{ml}, 100 \mathrm{mg} / \mathrm{ml}$ and $50 \mathrm{mg} / \mathrm{ml}$ ) were used. Paper discs of 0.3 diameter in size were impregnated with the selected plant extracts. It was left to dry for 10minutes, before dispensing unto the surface of the already inoculated Muller Hinton Agar /Sabouraud Dextrose Agar plate and allowed to diffuse for half an hour at $4^{0} \mathrm{C}$ on the surface of the medium. The plates were incubated at $37^{\circ} \mathrm{C}$ for 24 hours and the zone of inhibition around each disc was measured for sensitivity, mild sensitivity or resistance. Diameters of the inhibition zones were measured using a metre rule. The antibacterial activity was expressed as the mean zone of inhibition diameters $(\mathrm{mm})$ produced by the plant extract. Each assay was carried out in triplicates under strict aseptic conditions. The absence of zone inhibition was interpreted as the absence of activity (Kohner et al., 1994; Mathabe et al., 2006). The activities are expressed as resistant, if the zone of inhibition was less than $7 \mathrm{~mm}$, intermediate or mildly sensitive $(8-10 \mathrm{~mm}$ ) and sensitive if more than $11 \mathrm{~mm}$ (Assam et al., 2010).Commercially prepared antibiotics discs (Tetracycline, Ampicillin, Chloramphenicol and Ofloxacin) were used to determine the drug sensitivity and resistance pattern of bacteria. These discs were placed on the plates inoculated with different strains. These served as positive control. Disc containing extracting solvents served as negative control. Similar to this, fungicidal effect of the plant extract was assessed by the inhibition of mycelia growth of the fungus and was observed as a zone of inhibition near the disc. The antifungal effect was seen as crescent shaped zones of inhibition. Nystatin (standard antifungal agent) also served as its positive control.

Minimum Inhibitory Concentrations (MIC) and Minimum Bacteriocidal Concentration (MBC): This was carried out by broth dilution method and plating method according to European Society of Clinical Microbiology and Infectious Diseases (ESCMID, 2003) and 
(Andrews, 2006). Different concentrations of ethyl acetate and methanol extracts of leaves and peels of Carica papaya (Pawpaw) and Mangifera indica (Mango)from $400 \mathrm{mg} / \mathrm{ml}$ to $6.25 \mathrm{mg} / \mathrm{ml}$ $(400 \mathrm{mg} / \mathrm{ml}, 200 \mathrm{mg} / \mathrm{ml}, 100 \mathrm{mg} / \mathrm{ml}, 50 \mathrm{mg} / \mathrm{ml}, 25 \mathrm{mg} / \mathrm{ml}, 12.5 \mathrm{mg} / \mathrm{ml}, 6.25 \mathrm{mg} / \mathrm{ml})$ were prepared using extracting solvent following a two-fold dilution. Standardized test organisms in broth culture (Muiller Histone broth and Sabouraud Dextrose broth) were used. About 9ml of broth culture together with $0.5 \mathrm{ml}$ of standardized organism and $0.5 \mathrm{ml}$ of extracting solvent was placed in test tubes and incubated for 24 hours at $35^{\circ} \mathrm{C}$, the presence of turbidity indicated growth of organism while tube with less concentration, having no turbidity was recorded as MIC. This was done in triplicate. After determination of MIC, pour plate method was used to grow the test tubes positive to MIC. Plates were incubated for 24 hours at $35^{\circ} \mathrm{C}$. Plates showing no growth of organisms were taken as the MBC. Ampicillin and Nystatin served as positive control while the last column of test tubes was left blank (that is without extract or antibiotics). This served as negative control.

\section{RESULTS AND DISCUSSION Qualitative Phytochemical Analysis}

Table 1 shows the result obtained from the phytochemical screening of crude plant extract performed.It was observed that alkaloid, flavonoid, tannins were found present in ripe mango peel. Steroid was found to be present in mango leaves, unripe pawpaw peel and pawpaw leaves while phenol was found to be present only in mango leaves. Also mango leaves was found to contain all the compounds tested for (steroid, alkaloid, flavonoid, tannins, saponins, glycosides, anthraquinones, cardiac glycosides and phenol). Pawpaw leaves was also found to contain all compound except glycoside and phenol. Ripe pawpaw peel was found to contain only alkaloid and saponins while unripe pawpaw peel contained steroid, tannins and saponins. Also, Unripemango peel contained only alkaloid, flavonoids and saponins.

Table1: Qualitative Phytochemical screening of Plant Crude Extracts

\section{Bioactive}

Compounds

\section{Plant Extracts}

$\begin{array}{cccccc}\begin{array}{c}\text { Ripe mango } \\ \text { peel }\end{array} & \begin{array}{c}\text { Unripe } \\ \text { mango peel }\end{array} & \begin{array}{c}\text { Mango } \\ \text { leaves }\end{array} & \begin{array}{c}\text { Ripe } \\ \text { pawpaw peel }\end{array} & \begin{array}{c}\text { Unripe } \\ \text { pawpaw peel }\end{array} & \begin{array}{c}\text { Pawpaw } \\ \text { leaves }\end{array}\end{array}$

\begin{tabular}{|c|c|c|c|c|c|c|}
\hline Steroid & - & - & + & - & + & + \\
\hline Alkaloid & + & + & + & + & - & + \\
\hline Flavonoid & + & + & + & - & + & + \\
\hline Tannins & + & - & + & - & - & + \\
\hline Saponin & - & + & + & + & + & + \\
\hline Glycosides & - & - & + & - & - & - \\
\hline
\end{tabular}


Vol. 4, No. 05; 2019

ISSN: $2456-8643$

Anthraquinones

Cardiac

glycosides

Phenol

\section{Antimicrobial Activities of Plant Extracts on Selected Organism}

It was observed that ethyl acetate ripe mango peel extract was more active against Candida albicans with highest zone of inhibition of $18.3 \pm 5.1 \mathrm{~mm}$ while lowest zone of inhibition was observed with Pseudomonas aeruginosa at $10.0 \pm 2.6 \mathrm{~mm}$. Also, methanol Ripe mango peel extract had highest zone of inhibition against Escherichia coli at $20.7 \pm 2.5 \mathrm{~mm}$ while lowest zone of inhibition was observed against Candida albicans at $10.3 \pm 3.8 \mathrm{~mm}$.

It was observed that ethyl acetate Unripe mango peel extract was more active against Escherichia coli with highest zone of inhibition of $21.7 \pm 2.1 \mathrm{~mm}$ while lowest zone of inhibition was observed with Pseudomonas aeruginosaat $11.3 \pm 1.5 \mathrm{~mm}$. Also, methanol Unripe mango peel extract had highest zone of inhibition against Staphylococcus aureus at $22.3 \pm 3.2 \mathrm{~mm}$ while lowest zone of inhibition was observed with Candida albicans at $10.3 \pm 5.0 \mathrm{~mm}$.

It was observed that both ethyl acetate and methanol Mango leaves extract were active against Staphylococcus aureus with highest zone of inhibition of $22.3 \pm 1.2 \mathrm{~mm}$ and $23.7 \pm 2.1 \mathrm{~mm}$ respectively while lowest zone of inhibition for ethyl acetate Mango leaves was observed against Escherichia coli at $11.0 \pm 1.7 \mathrm{~mm}$ and for methanol Mango leaves extract, it was observed to have lower zone of inhibition against Candida albicans at $15.3 \pm 12.5 \mathrm{~mm}$.

It was observed that both ethyl acetate and methanol Ripe Pawpaw peel extract were active against Staphylococcus aureuswith highest zone of inhibition of $14.3 \pm 9.3 \mathrm{~mm}$ and $22.7 \pm$ $7.2 \mathrm{~mm}$ respectively. Lowest zone of inhibition for ethyl acetateRipe Pawpaw peel was observed against Escherichia coli at $10.0 \pm 4.4 \mathrm{~mm}$. Also for methanol Ripe Pawpaw peel extract was observed to have lower zone of inhibition against Candida albicans at $14.7 \pm 9.3 \mathrm{~mm}$.

It was observed that ethyl acetate Unripe Pawpaw peel extract was more active against Pseudomonas aeruginosa with highest zone of inhibition of $21.7 \pm 3.1 \mathrm{~mm}$ while it lowest zone of inhibition was observed against Candida albicans at $10.0 \pm 2.0 \mathrm{~mm}$. Also, methanol Unripe Pawpaw peel extract had highest zone of inhibition against Staphylococcus aureusat $19.0 \pm$ $6.1 \mathrm{~mm}$ while its lowest zone of inhibition was observed against Candida albicans at $10.7 \pm$ $3.1 \mathrm{~mm}$.

It was observed that ethyl acetate Pawpaw leaves extract was more active against Escherichia coliwith highest zone of inhibition of $27.3 \pm 11 . \mathrm{mm}$ while its lowest zones of inhibition was against Staphylococcus aureus at $18.7 \pm 3.2 \mathrm{~mm}$. Methanol Pawpaw leaves extract had highest zone of inhibition against Staphylococcus aureusat $25.0 \pm 13.0 \mathrm{~mm}$ while lowest zones of 
inhibition was observed against Escherichia coli and Candida albicans at $12.0 \pm 10.6 \mathrm{~mm}$ and $12.0 \pm 10.4 \mathrm{~mm}$ respectively

Furthermore, the varied concentrations $(200 \mathrm{mg} / \mathrm{ml})$ of ethyl acetate Unripe mango peel, gave zones of inhibition sensitive against Staphylococcus aureus at 12.7 \pm 4.5 and Candida albicans at 12.3 \pm 7.4. Also, mango leaves showed sensitive zones of inhibition against Staphylococcus aureusat 15.3 \pm 0.6 andCandida albicans at 11.0 \pm 4.6 , and pawpaw leaves showed sensitive zones of inhibition against all four organisms (Escherichia coli, Staphylococcus aureus, Pseudomonas aeruginosa and Candida albicans) at $12.7 \pm 2.1,10.7 \pm 1.2,16.7 \pm 1.5$ and $14.0 \pm 2.6$ respectively. All other concentrations showed intermediate to non-sensitive zones of inhibition. Whereas, varied concentrations $(200 \mathrm{mg} / \mathrm{ml})$ of methanol extract ripe mango peel gave sensitive zones of inhibition against Escherichia coli at 13.7 \pm 5.0 , unripe mango peel showed sensitive zones of inhibition against Escherichia coli and Staphylococcus aureus, at 13.3 \pm 5.5 and 13.0 \pm 5.6 respectively, mango leaves showed sensitive zones of inhibition against Escherichia coli and Staphylococcus aureus, at $16.7 \pm 4.2$ and $14.3 \pm 4.7$ respectively, ripe pawpaw peel showed sensitive zones of inhibition against Staphylococcus aureus, at 12.3 \pm 4.5 and pawpaw leaves

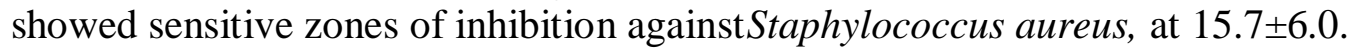

\begin{tabular}{|c|c|c|c|c|c|c|c|c|c|}
\hline $\begin{array}{c}\text { Plant } \\
\text { Extract }\end{array}$ & \multirow{3}{*}{ Organism } & \multicolumn{8}{|c|}{ Concentration( $(\mathrm{mg} / \mathrm{ml}) /$ Average Zone of Inhibition(mm) } \\
\hline \multirow{6}{*}{$\begin{array}{c}\text { RIPE } \\
\text { MANGO } \\
\text { PEEL }\end{array}$} & & \multicolumn{4}{|c|}{ Ethyl acetate } & \multicolumn{4}{|c|}{ Methanol } \\
\hline & & 400 & 200 & 100 & $\mathbf{5 0}$ & \multirow{2}{*}{$\begin{array}{c}\mathbf{4 0 0} \\
20.7 \pm 2.5\end{array}$} & \multicolumn{2}{|c|}{\begin{tabular}{|l|l}
200 & 100 \\
\end{tabular}} & $\mathbf{5 0}$ \\
\hline & $\begin{array}{l}\text { Escherichia } \\
\text { coli }\end{array}$ & $13.7 \pm 4.9$ & - & - & - & & $\begin{array}{l}13.7 \pm 5 \\
.0\end{array}$ & $\begin{array}{l}10.7 \pm 4 \\
.2\end{array}$ & $\begin{array}{l}6.3 \pm 2 \\
.1\end{array}$ \\
\hline & $\begin{array}{c}\text { Staphylococcus } \\
\text { aureus }\end{array}$ & $17.3 \pm 3.1$ & $\begin{array}{c}9.3 \pm 1 \\
5\end{array}$ & $\begin{array}{l}3.0 \pm 1 \\
.7\end{array}$ & $\begin{array}{l}2.0 \pm 1 . \\
0\end{array}$ & $22.0 \pm 5.3$ & $\begin{array}{l}9.7 \pm 7 \\
7\end{array}$ & $\begin{array}{l}9.7 \pm 2 . \\
9\end{array}$ & $\begin{array}{l}4.7 \pm 1 \\
.5\end{array}$ \\
\hline & $\begin{array}{l}\text { Pseudomonas } \\
\text { aeruginosa }\end{array}$ & $10.0 \pm 2.6$ & - & - & - & $13.7 \pm 3.1$ & - & - & - \\
\hline & $\begin{array}{l}\text { Candida } \\
\text { albicans }\end{array}$ & $18.3 \pm 5.1$ & $\begin{array}{c}9.3 \pm 3 \text {. } \\
5\end{array}$ & $\begin{array}{l}6.0 \pm 1 \\
.0\end{array}$ & $\begin{array}{l}2.7 \pm 0 . \\
6\end{array}$ & $10.3 \pm 3.8$ & - & - & - \\
\hline
\end{tabular}




\section{International Journal of Agriculture, Environment and Bioresearch}

Vol. 4, No. 05; 2019

ISSN: $2456-8643$

\begin{tabular}{|c|c|c|c|c|c|c|c|c|c|}
\hline \multirow{4}{*}{$\begin{array}{c}\text { UNRIPE } \\
\text { MANGO } \\
\text { PEEL }\end{array}$} & $\begin{array}{l}\text { Escherichia } \\
\text { coli }\end{array}$ & $21.7 \pm 2.1$ & $\begin{array}{c}10.0 \pm 1 \\
.0\end{array}$ & $\begin{array}{l}6.0 \pm 1 \\
.0\end{array}$ & $\begin{array}{l}2.3 \pm 1 \\
2\end{array}$ & $21.3 \pm 3.1$ & $\begin{array}{l}13.3 \pm 5 \\
.5\end{array}$ & $\begin{array}{l}8.7 \pm 3 . \\
8\end{array}$ & $\begin{array}{l}4.3 \pm 3 \\
.5\end{array}$ \\
\hline & $\begin{array}{c}\text { Staphylococcus } \\
\text { aureus }\end{array}$ & $20.0 \pm 2.6$ & $\begin{array}{c}12.7 \pm 4 \\
.5\end{array}$ & $\begin{array}{l}6.3 \pm 0 \\
.6\end{array}$ & $\begin{array}{l}3.3 \pm 0 . \\
6\end{array}$ & $22.3 \pm 3.2$ & $\begin{array}{l}13.0 \pm 5 \\
.6\end{array}$ & $\begin{array}{l}7.0 \pm 2 . \\
0\end{array}$ & $\begin{array}{l}1.3 \pm 1 \\
.5\end{array}$ \\
\hline & $\begin{array}{l}\text { Pseudomonas } \\
\text { aeruginosa }\end{array}$ & $11.3 \pm 1.5$ & - & - & - & $14.3 \pm 1.5$ & - & - & - \\
\hline & $\begin{array}{l}\text { Candida } \\
\text { albicans }\end{array}$ & $\begin{array}{l}17.7 \pm 10 . \\
2\end{array}$ & $\begin{array}{c}12.3 \pm 7 \\
.4\end{array}$ & $\begin{array}{l}6.3 \pm 2 \\
.3\end{array}$ & $\begin{array}{l}1.3 \pm 2 \\
3\end{array}$ & $10.3 \pm 5.0$ & - & - & - \\
\hline \multirow{4}{*}{$\begin{array}{l}\text { MANGO } \\
\text { LEAVES }\end{array}$} & $\begin{array}{l}\text { Escherichia } \\
\text { coli }\end{array}$ & $11.0 \pm 1.7$ & - & - & - & $22.7 \pm 0.6$ & $\begin{array}{l}16.7 \pm 4 \\
.2\end{array}$ & $\begin{array}{l}9.3 \pm 4 . \\
2\end{array}$ & $\begin{array}{l}5.3 \pm 2 \\
.1\end{array}$ \\
\hline & $\begin{array}{c}\text { Staphylococcus } \\
\text { aureus }\end{array}$ & $22.3 \pm 1.2$ & $\begin{array}{l}15.3 \pm 0 \\
.6\end{array}$ & $\begin{array}{l}8.7 \pm 1 \\
.5\end{array}$ & $\begin{array}{l}3.7 \pm 0 . \\
6\end{array}$ & $23.7 \pm 2.1$ & $\begin{array}{l}14.3 \pm 4 \\
.7\end{array}$ & $\begin{array}{l}6.7 \pm 3 . \\
1\end{array}$ & $\begin{array}{l}5.3 \pm 2 \\
.5\end{array}$ \\
\hline & $\begin{array}{l}\text { Pseudomonas } \\
\text { aeruginosa }\end{array}$ & $15.7 \pm 1.5$ & $\begin{array}{c}9.0 \pm 1 . \\
0\end{array}$ & $\begin{array}{l}4.0 \pm 1 \\
.0\end{array}$ & $\begin{array}{l}1.3 \pm 0 \\
5\end{array}$ & $18.3 \pm 6.7$ & $\begin{array}{l}9.7 \pm 9 . \\
5\end{array}$ & $\begin{array}{l}9.3 \pm 3 . \\
2\end{array}$ & $\begin{array}{l}2.0 \pm 1 \\
.7\end{array}$ \\
\hline & $\begin{array}{l}\text { Candida } \\
\text { albicans }\end{array}$ & $18.3 \pm 5.7$ & $\begin{array}{l}11.0 \pm 4 \\
.6\end{array}$ & $\begin{array}{l}5.0 \pm 4 \\
.4\end{array}$ & $\begin{array}{l}1.7 \pm 0 . \\
6\end{array}$ & $\begin{array}{l}15.3 \pm 12 . \\
5\end{array}$ & $\begin{array}{l}6.7 \pm 6 \\
1\end{array}$ & $\begin{array}{l}4.3 \pm 3 . \\
2\end{array}$ & $\begin{array}{l}1.7 \pm 2 \\
.1\end{array}$ \\
\hline \multirow{3}{*}{$\begin{array}{c}\text { RIPE } \\
\text { PAWPAW } \\
\text { PEEL }\end{array}$} & $\begin{array}{c}\text { Escherichia } \\
\text { coli }\end{array}$ & $10.0 \pm 4.4$ & - & - & - & $15.3 \pm 2.1$ & $\begin{array}{l}8.7 \pm 2 . \\
1\end{array}$ & $\begin{array}{l}5.0 \pm 2 . \\
0\end{array}$ & $\begin{array}{l}2.7 \pm 1 \\
.5\end{array}$ \\
\hline & $\begin{array}{c}\text { Staphylococcus } \\
\text { aureus }\end{array}$ & $14.3 \pm 9.3$ & - & - & - & $22.7 \pm 7.2$ & $\begin{array}{l}12.3 \pm 4 \\
.5\end{array}$ & $\begin{array}{l}7.7 \pm 2 . \\
1\end{array}$ & $\begin{array}{l}5.7 \pm 3 \\
.1\end{array}$ \\
\hline & $\begin{array}{l}\text { Pseudomonas } \\
\text { aeruginosa }\end{array}$ & $10.7 \pm 9.3$ & - & - & - & $17.7 \pm 6.0$ & $\begin{array}{l}10.3 \pm 4 \\
.0\end{array}$ & $\begin{array}{l}5.3 \pm 2 . \\
5\end{array}$ & $\begin{array}{l}2.7 \pm 1 \\
.5\end{array}$ \\
\hline
\end{tabular}


International Journal of Agriculture, Environment and Bioresearch

Vol. 4, No. 05; 2019

ISSN: $2456-8643$

\begin{tabular}{|c|c|c|c|c|c|c|c|c|c|}
\hline & $\begin{array}{l}\text { Candida } \\
\text { albicans }\end{array}$ & $\begin{array}{l}13.0 \pm 11 . \\
3\end{array}$ & - & - & - & $14.7 \pm 9.3$ & - & - & - \\
\hline \multirow{4}{*}{$\begin{array}{c}\text { UNRIPE } \\
\text { PAWPAW } \\
\text { PEEL }\end{array}$} & $\begin{array}{l}\text { Escherichia } \\
\text { coli }\end{array}$ & $\begin{array}{l}11.7 \pm 10 . \\
2\end{array}$ & - & - & - & $16.3 \pm 4.0$ & $\begin{array}{l}7.3 \pm 7 . \\
5\end{array}$ & $\begin{array}{l}4.7 \pm 3 . \\
8\end{array}$ & $\begin{array}{l}1.7 \pm 2 \\
.1\end{array}$ \\
\hline & $\begin{array}{c}\text { Staphylococcus } \\
\text { aureus }\end{array}$ & $12.7 \pm 4.0$ & - & - & - & $19.0 \pm 6.1$ & $\begin{array}{l}9.7 \pm 2 . \\
5\end{array}$ & $\begin{array}{l}5.7 \pm 3 . \\
5\end{array}$ & $\begin{array}{l}2.3 \pm 2 \\
.1\end{array}$ \\
\hline & $\begin{array}{l}\text { Pseudomonas } \\
\text { aeruginosa }\end{array}$ & $21.7 \pm 3.1$ & $\begin{array}{c}9.0 \pm 7 \\
3\end{array}$ & $\begin{array}{l}7.7 \pm 1 \\
.2\end{array}$ & $\begin{array}{l}3.7 \pm 1 \\
5\end{array}$ & $13.3 \pm 6.7$ & - & - & - \\
\hline & $\begin{array}{l}\text { Candida } \\
\text { albicans }\end{array}$ & $10.0 \pm 2.0$ & - & - & - & $10.7 \pm 3.1$ & - & - & - \\
\hline \multirow{4}{*}{$\begin{array}{c}\text { PAWPAW } \\
\text { LEAVES }\end{array}$} & $\begin{array}{l}\text { Escherichia } \\
\text { coli }\end{array}$ & $\begin{array}{l}27.3 \pm 11 . \\
7\end{array}$ & $\begin{array}{c}12.7 \pm 2 \\
.1\end{array}$ & $\begin{array}{l}7.7 \pm 2 \\
.5\end{array}$ & - & $\begin{array}{l}12.0 \pm 10 . \\
6\end{array}$ & - & - & - \\
\hline & $\begin{array}{c}\text { Staphylococcus } \\
\text { aureus }\end{array}$ & $18.7 \pm 3.2$ & $\begin{array}{c}10.7 \pm 1 \\
.2\end{array}$ & $\begin{array}{l}7.3 \pm 0 \\
.6\end{array}$ & $\begin{array}{l}1.7 \pm 1 \\
2\end{array}$ & $\begin{array}{l}25.0 \pm 13 . \\
0\end{array}$ & $\begin{array}{l}15.7 \pm 6 \\
.0\end{array}$ & $\begin{array}{l}9.3 \pm 3 . \\
2\end{array}$ & - \\
\hline & $\begin{array}{l}\text { Pseudomonas } \\
\text { aeruginosa }\end{array}$ & $\begin{array}{l}21.7 \pm 11 . \\
8\end{array}$ & $\begin{array}{c}16.7 \pm 1 \\
.5\end{array}$ & $\begin{array}{l}6.3 \pm 1 \\
.5\end{array}$ & $\begin{array}{l}2.7 \pm 0 \\
6\end{array}$ & $15.0 \pm 8.7$ & $\begin{array}{l}10.3 \pm 9 \\
.1\end{array}$ & $\begin{array}{l}4.3 \pm 2 . \\
1\end{array}$ & $\begin{array}{l}3.3 \pm 1 \\
.2\end{array}$ \\
\hline & $\begin{array}{l}\text { Candida } \\
\text { albicans }\end{array}$ & $25.3 \pm 0.6$ & $\begin{array}{c}14.0 \pm 2 \\
.6\end{array}$ & $\begin{array}{l}6.3 \pm 2 \\
.3\end{array}$ & - & $\begin{array}{l}12.0 \pm 10 . \\
4\end{array}$ & - & - & - \\
\hline
\end{tabular}

Antibiotics Sensitivity testing against Clinical Isolates: Using Tetracycline, Ampicillin, Ofloxacin, Chloramphenicol (antibiotics) as positive control for antibacterial analysis and Nystatin (antifungal) as positive control for antifungal analysis against isolatesrevealed that for Escherichia coli, tetracycline, ampicillin, ofloxacin and chloramphenicol gave zones of inhibition of $17.0 \pm 0.00 \mathrm{~mm}, 25.0 \pm 0.00 \mathrm{~mm}, 28.0 \pm 0.00 \mathrm{~mm}$ and $20.0 \pm 0.00 \mathrm{~mm}$ respectively. For Staphylococcus aureus, tetracycline, ampicillin, ofloxacin and chloramphenicol gave zones 
of inhibition of $20.0 \pm 0.00 \mathrm{~mm}, 18.0 \pm 0.00 \mathrm{~mm}, 24.0 \pm 0.00 \mathrm{~mm}$ and $23.0 \pm 0.00 \mathrm{~mm}$ respectively. For Pseudomonas aeruginosa, tetracycline, ampicillin, ofloxacin and chloramphenicol gave zones of inhibition of $20.0 \pm 0.00 \mathrm{~mm}, 23.0 \pm 0.00 \mathrm{~mm}, 22.0 \pm 0.00 \mathrm{~mm}$ and $12.0 \pm 0.00 \mathrm{~mm}$ respectively. For Candida albicans, nystatin gave zone of inhibition of $26.0 \pm 0.00 \mathrm{~mm}$. Negative control using extracting solvent was negative for both ethyl acetate and methanol.

Table 3: Antibiotics Sensitivity testing against Clinical Isolates

\begin{tabular}{|c|c|c|c|c|c|c|c|}
\hline \multicolumn{6}{|c|}{ Positive control (mm) } & \multicolumn{2}{|c|}{ Negative control } \\
\hline & & & & Chloram & & Ethyl & \\
\hline Organisms & Tetracycline & Ampicillin & Ofloxacin & phenicol & Nystatin & Acetate & Methanol \\
\hline Escherichia coli & 17.0 & 25.0 & 28.0 & 20.0 & - & & \\
\hline $\begin{array}{c}\text { Staphylococcus } \\
\text { aureus }\end{array}$ & 20.0 & 18.0 & 24.0 & 23.0 & - & & \\
\hline $\begin{array}{l}\text { Pseudomonas } \\
\text { aeruginosa }\end{array}$ & 20.0 & 23.0 & 22.0 & 12.0 & - & & \\
\hline $\begin{array}{l}\text { Candida } \\
\text { albicans }\end{array}$ & - & - & - & - & 26.0 & & \\
\hline & & & & & & Negative & Negative \\
\hline
\end{tabular}

\section{Minimum Inhibitory and Bactericidal Concentration of Plant Extract on Microbial Isolates}

The minimum inhibitory concentration (MIC) values of Unripe mango peel and Mango leaves ethyl acetate extracts treated on $S$. aureus and Mango leaves ethyl acetate extracts against $C$. albicans was found to be $200 \mathrm{mg} / \mathrm{ml}$. On the other hand, the MIC values of Pawpaw leaves ethyl acetate extract treated on E. coli and $P$. aeruginosa were found to be $100 \mathrm{mg} / \mathrm{ml}$ and $200 \mathrm{mg} / \mathrm{ml}$ respectively. For Mango and Pawpaw leaves, methanol extract showed MIC values against $S$. aureus at $200 \mathrm{mg} / \mathrm{ml}$ and $100 \mathrm{mg} / \mathrm{ml}$ respectively, while Mango and Pawpaw leaves methanol extracts showed MIC values against $P$. aeruginosa at $100 \mathrm{mg} / \mathrm{ml}$ respectively. Unripe mango peel was observed to have shown MIC value when used against $E$. coli at $100 \mathrm{mg} / \mathrm{ml}$. MIC values of Nystatin and ampicillin was determined on C. albicans, E. coli and S. aureus and showed results of $50 \mathrm{mg} / \mathrm{ml}$ and $100 \mathrm{mg} / \mathrm{ml}$ respectively. Negative control was left blank and therefore showed no results.

Minimum bactericidal concentration of plant extracts of unripe mango peels, mango leavesethyl acetate extract and unripe mango peels, mango and pawpaw leaves methanol extract showed further growth of microorganisms at $200 \mathrm{mg} / \mathrm{ml}$ concentration. However pawpaw leaves ethyl acetate extracts of $100 \mathrm{mg} / \mathrm{ml}$ and $200 \mathrm{mg} / \mathrm{ml}$ concentrations respectively against $E$. coliand $P$. aeruginosa showed no further growth on petri dish. 
Table 4: Minimum Inhibitory Concentrations of Plant Extracts

\begin{tabular}{|llllll|}
\hline Extracting & Plant & \multicolumn{4}{l}{ Minimum Inhibitory Concentrations (mg/ml) } \\
\cline { 3 - 6 } & Extract & E. coli & S. aureus & P. aeruginosa & C. albicans \\
\hline \multirow{2}{*}{ Ethyl Acetate } & UMP & - & 200 & - & - \\
& ML & - & 200 & - & 200 \\
& PL & 100 & - & 200 & - \\
Methanol & UMP & 200 & - & - & - \\
& ML & - & 200 & 100 & - \\
Nystatin & PL & - & 100 & 100 & - \\
(positive control) & & - & & & 50 \\
$\begin{array}{l}\text { Ampicillin } \\
\text { (positive control) }\end{array}$ & & 100 & 100 & - & - \\
& & & & - & \\
\hline
\end{tabular}

UMP- Unripe mango peel, ML- Mango leaves, PL- Pawpaw leaves

\section{DISCUSSION}

Phytochemical screening: The results obtained from this study which carried out an analysis of the qualitative phytochemical properties of crude extracts of some selected plant samples against some microbial isolates using six plant samples (Mango leaves, Ripe and Unripe mango peels, Pawpaw leaves, and Ripe and Unripe pawpaw peels) showed the presence of phytochemical properties. Similar findings were made byAyoola and Adeyeye (2010) who in their findings on the phytochemical properties of the leaves of Carica papaya showed the presence of saponins, cardiac glycosides, and alkaloids. It is also in agreement with the findings of Okwu and Ezenagu (2008), Aravindet al., (2013) and Vijayakumar et al., (2015). The presence of saponins according to Okwu and Okwu. (2004), indicates the cytotoxic effects of the plant extracts. The presence of the bioactive components in pawpaw and mango leaves extract according to Mary et al., (2013) andAravind et al., (2013), could be said to be the reasons of its effectiveness against microorganisms.

Antimicrobial activities of plant extracts on selected isolates: According to Bacon et al., (2017), the higher the concentration of extract to solvent the more the zone of inhibition. This therefore supports the reason for the observed higher zones of inhibition in higher concentrations in this research. Zones of inhibition $\leq 7 \mathrm{~mm}$ indicated that the organism was resistant, $8-10 \mathrm{~mm}$ indicated an intermediate sensitivity, while $\geq 11 \mathrm{~mm}$ indicated sensitivity. Sensitivity implied that the plant 
extract could inhibit the growth of that particular organism at the given level of concentration. $400 \mathrm{mg} / \mathrm{ml}$ and $200 \mathrm{mg} / \mathrm{ml}$ concentrations gave the best results in both extracting solvent. For ethyl acetate extracts, Pawpaw leaves gave the best results with highest zones of inhibition as can be observed in table 2 against Escherichia coli and Candida albicans respectively. Meanwhile, in methanol extracts, Pawpaw leaves gave the best result with highest zone of inhibition against Staphylococcus aureus. Mango leaves was also observed to have given the high zones of inhibition against Staphylococcus aureus as seen in Table 2. This result shows that pawpaw and mango leaves plant extract is active against both gram positive and gram negative bacteria therefore is at disparity with an earlier report by Jigna and Chanda (2006), who in his findings indicated that plant extracts were more active against gram-positive bacteria than gram-negative bacteria.

Antibiotic sensitivity testing had zones of inhibition equivalent to that of plant part extracts. This therefore suggests that plant parts especially pawpaw and mango leaves extract are as good as other commercially sold antibiotics in inhibiting these microorganisms and therefore could possibly serve as an alternative. Pawpaw leaves had no further growth and was also taken as the minimal bactericidal concentration.

\section{CONCLUSION}

Based on the findings from the study it can be concluded that: Plant samples contained several bioactive compounds (steroid, alkaloid, flavonoid, tannins, saponins, glycosides, anthraquinones, cardiac glycosides and phenol) which accounted for the activities of plant against microorganisms. The minimum bactericidal concentration was found with ethyl acetate pawpaw leaves extract against Escherichia coli and Pseudomonas aeruginosa (bactericidal) while all the others were bacteriostatic.Pawpaw leaves was found to have the highest zone of inhibition and could therefore be said to be the best plant extract against the selected clinical bacterial isolates.

\section{REFERENCES}

Alonso-Paz, E., Cerdeiras, M.P., Fernandez, J., Ferreira, F., Moyna, P., Soubes, M., Vazquez, A., Veros, S. and Zunno, L. (1995). Screening of Uruguayan medicinal plants for antimicrobial activity. J. Ethnopharmacology.45: 67-70.

Anesini, E.and Perez, C. (1993). Screening of plants used in Argentine folk medicine for antimicrobial activity. J. Ethnopharmacol.39: 119-128.

Assam, J.P.,Dzoyem, J.P., Pieme, C.A. and Penlap, V.B. (2010).In vitro antibacterial activity and acute toxicity studies of aqueous-methanol extract of Sidarhombifolia Linn. (Malvaceae). Journal of the International Society for Complementary Medicine Research (ISCMR) 10:40

Bacon, K., Boyer, R., Denbow, C., O’keefe, S., Neilson, A. and Williams, R. (2017). Evaluation of different solvents to extract antibacterial compounds. Food SciNutr. 5: 497-530.

Barreto, J.C., Trevisan, M.T. and Hull, W. E. (2008). Characterization and quantitation of polyphenolic compounds in bark, kernel, leaves, and peel of mango (Mangiferaindica L.).J. Agric. Food Chem.56(14): 5599-5610. 
Bruna, E.P., Fernandes, B., Borges, A.C., Almeida, J. and Barros, N.F. (1989). Effects of Eucalyptus litter extracts on microbial growth. Pesq. Agrop. Bras.24: 1523-1528.

Carvalho, V., Melo, V.M., Aguiar, A. and Matos, F.S. (1988). Toxicity evaluation of medicinal plant extracts by the brine shrimp (Arthenussalina Leah) biossay. Ciência e Cultura40: 11091111.

Cohen, M.L. (1992). Epidemiology of drug resistance. In Implications for a post-antimicrobial era. Science.257: 1050-1055.

Cruz, F.G., Roque, N.F., Giesbrecht, A.M.andDavino, S.C. (1996). Antibiotic activity of diterpenes from Mikaniatriangularis._Fitoterapia. 67:189-190.

Donatus, E. O. and Vitus, E. (2008). Evaluation of the phytochemical composition of mango (mangiferaindicalinn) stem bark and leaves. Int. J. Chem. Sci.6(2): 705-716

Kohner, P.C., Rosenblatt, J.E. and Cockerill, F.R. (1994). Comparison of agar dilution, broth dilution and disk diffusion testing of Ampicillin against Haemophilus spp. by using in house and commercially prepared media. J. Clin. Microbiol. 32: 1594 -96.

Lemos, T.L.G., Monte, F.J.Q., Matos, F.J.A., Alencar, J.W., Craveiro, A.A., Barbosa, R.C.S.B. and Lima, E.D. (1992). Chemical composition and antimicrobial activity of essencial oils from Brazilian plants. Fitoterapia.63: 266-268.

Martinez, M.J., Betancourt, J., Alonso-Gonzalez, N. and Jauregui, A. (1996). Screening of some Cuban medicinal plants for antimicrobial activity. J. Ethnopharmacol.52: 171-174.

Martinez, M.J., Vasquez, S.M., Espinosa-Perez, C., Dias, M. and Herrera-Sanchez, M.(1994). Antimicrobial properties of Argentatine A isolated from Partheniumargentatum. Fitoterapia.65: 371-372.

Martins, E. (2014).The growing use of herbal medicines: issues relating to adverse reaction and challenges in monitoring safety. FrontiersinPharmacology. 4: 170.

Mary- Helen, P.A., Aswathy, M.R., Deepthi, K.G., RathiMol, R., Jaison, J. J. and Jaya, S. S. (2013). Phytochemical analysis and anticancer activity of leaf extract of mangiferaindica (kottukonamvarika).International Journal Of Pharmaceutical Sciences And Research. Pp823828.

Mathabe, M.C., Nikolova, R.V., Lall, N. and Nyazema, N.Z.(2006). Antibacterial activities of medicinal plants used for the treatment of diarrhea in Limpopo Province, South Africa. JournalofEthnopharmacology.105:286-293.

Matos, F.J.A., Aguiar, L.M.B.A. and Silva, M.G.A. (1988). Chemical constituents and antimicrobial activity of VataireamacrocarpaDucke. ActaAmazonica. 18: 351-352.

Nascimento, S.C., Chiappeta, A. and Lima, R.M.O.C. (1990). Antimicrobial and cytotoxic activities in plants from Pernambuco, Brazil. Fitoterapia.61:353-355. 
Nunez-Selles, J. (2005). Antioxidant Therapy; Myth or Reality. J. Braz. Chem. Soc.16(4): 101 108.

Nwankwo, I.U. and Osaro-Mathew, R.C. (2014). Assessment of the phytochemical components of Mangiferaindica(leaf) and Musa paradisiaca (roots) extracts and their antibacterial activity against some common pathogenic bacteria. Journal of Pharmacy and Biological Sciences. 9: 0811

Nweze, L.A., Okafor, J.L. and Nwoku, O. (2004). Antimicrobial Activities ofMethanolic extracts of Tremeguineenes (Schumm and thom) and MorindalucidaBenth used in Nigeria traditional herbal medicinal practice. Biol. Res. 2: 33-48.

Santos Filho, D., Sarti, S.J., Bastos, J.K., LeitãoFilho, H.F., Machado, J.O., Araujo, M.L.C., Lopes, W.D. and Abreu, J.E. (1990). Atividadeantibacteriana de extratosvegetais. Rev. Cien. Farm.12: 39-46.

Sanusi, B. M., Auwalu, G., Aliyu, M., Aminu, M. and David, O.A. (2012). Phytochemical Screening and Antimicrobial Efficacy of Aqueous and Methanolic Extract of Mangiferaindica (Mango Stem Bark).World J Life Sci. and Medical Research. 2(2): 81-85

Senthilkumar, P.K. and Reetha, D. (2009). Regular article Screening of antimicrobial properties of certain Indian medicinal plants. Journal of Phytology. 1(3): 193-198

Sofowora, A.O. (1993). Medicinal Plants and Traditional Medicine in Africa. University of Ife Press 2nd Ed. Pp 320.

Subramanian, G., Brij, B.T., Rekha, G. (2014). Antimicrobial properties of Carica papaya(papaya) different leaf extract against E. coli, S. aureus, C. albicans. American Journal of Pharmacology and Pharmacotherapeutics. 1: 25-39. 